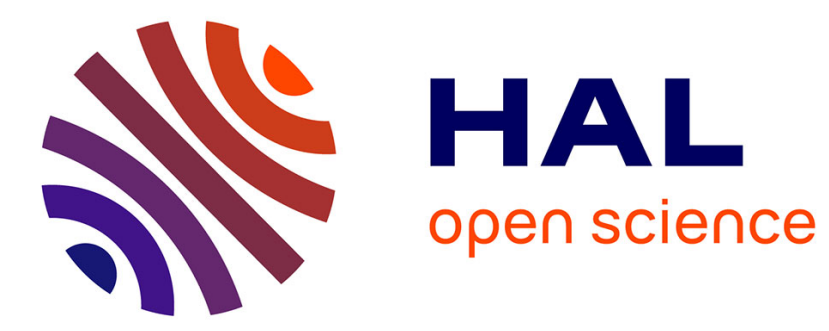

\title{
Les Religieuses en mouvement. Ursulines françaises et Dames anglaises à l'aube du XVIIe siècle \\ Laurence Lux-Sterritt
}

\section{To cite this version:}

Laurence Lux-Sterritt. Les Religieuses en mouvement. Ursulines françaises et Dames anglaises à l'aube du XVIIe siècle. Revue d'Histoire Moderne et Contemporaine, 2005, 52 (4), pp. 7-23. hal-01060743

\section{HAL Id: hal-01060743 \\ https://hal.science/hal-01060743}

Submitted on 4 Sep 2014

HAL is a multi-disciplinary open access archive for the deposit and dissemination of scientific research documents, whether they are published or not. The documents may come from teaching and research institutions in France or abroad, or from public or private research centers.
L'archive ouverte pluridisciplinaire HAL, est destinée au dépôt et à la diffusion de documents scientifiques de niveau recherche, publiés ou non, émanant des établissements d'enseignement et de recherche français ou étrangers, des laboratoires publics ou privés. 


\section{Les Religieuses en mouvement. Ursulines françaises et Dames anglaises à l'aube du XVII ${ }^{\mathrm{e}}$ siècle.}

Laurence Lux-Sterritt

"Une religieuse hors de sa clôture est . . . comme un arbre hors de terre ; ... comme un poisson hors de l'eau ...; comme une brebis hors de sa bergerie et en danger d'être dévorée des loups ... et par conséquent dans un état tout à fait opposé à

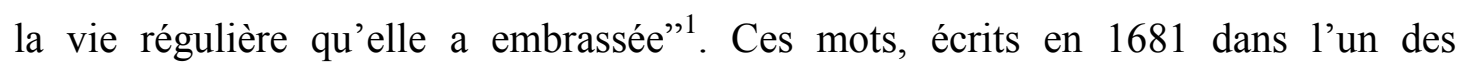
nombreux traités du genre qui paraissent à cette époque, trahissent un questionnement profond quant à la nature de la femme d'Église et plus particulièrement quant à sa relation avec la liberté de mouvement. Le $X V I^{\mathrm{e}}{ }^{\mathrm{s}}$ siècle marque en effet une ère nouvelle pour le catholicisme, un renouveau qui se caractérise entre autres par une certaine "féminisation de l'Église"². Sur le sol français, l'essor est omniprésent : partout se créent des salons dévots, partout de nouvelles écoles qui, pour contrecarrer les établissements protestants, se consacrent désormais à l'instruction religieuse des filles ainsi que des garçons, inscrivant la femme au cœur même de l'impulsion de la contre-réforme ${ }^{3}$. Dans l'Angleterre protestante et malgré la clandestinité à laquelle il est condamné, le renouveau catholique se manifeste par le soubresaut d'énergie qui ranime alors la communauté récusante. Tandis que les prêtres missionnaires et les Jésuites en particulier entreprennent de reconvertir leur terre natale, ils sont aidés par la participation de femmes sans qui la mission n'aurait su connaître le succès qu'on lui reconnaît ${ }^{4}$.

\footnotetext{
1. Jean Bâptiste THIERs, Traité de la clôture des religieuses, Paris, Dezallier, 1681, p.243.

2. Elizabeth RAPLEY, The Dévotes : Women and Church in Seventeenth-Century France, Kinston, Ont, McGill, Queen's University Press, 1990, p.193-6.

3. Voir entre autre les travaux de Henri BRÉMOND, Histoire littéraire du sentiment religieux en France depuis la fin des guerres de religion jusqu'à nos jours, 11 vols, Paris, A. Colin, 1971 ; Pierre Chaunu, L'Église, culture et société : essai sur réforme et contre-réforme, Paris, SEDES, 1984 et René TAVENEAUX, Le Catholicisme dans la France classique, 1610-1717, 2 vols, Paris, SEDES, 1980.

${ }^{4}$. Certaines études se penchent sur le rôle actif joué par les femmes dans l'Angleterre récusante ; voir entre autres John Bossy, The English Catholic Community, 1570-1850, Londres, Darton, Longman and Todd, 1976 ; Roland Connelly, Women of the Catholic Resistance in England 1540-1680, Durham, Pentland Press, 1997 ; Alexandra Walsham, Church Papists: Catholicism, Conformity and Confessional Polemic in Early Modern England, Woodbridge, Boydell Press, 1993 et Marie Rowlands, 'Recusant Women 1560-1640', in Mary PrIOR (dir.) Women in English Society 1500-1800, Londres, Methuen, 1985.
} 
Cet élan de reconquête nécessite bien sûr l'action et la mobilité physique. Or, si le concile de Trente (1545-1563) accorde aux hommes d'Église le droit de servir une vocation apostolique dans le siècle, il impose au contraire la clôture aux congrégations féminines : en 1566, la bulle Circa Pastoralis de Pie V renforce la décrétale Periculoso de 1299 et réaffirme du même coup les traditions médiévales qui n'offrent à la religieuse d'autre alternative que le couvent. Le décret est catégorique : “s'il se trouvait par hasard des religieuses qui, s'appuyant sur quelque coutume, même immémoriale ... s'opposassent par opiniâtreté à cette clôture ... les ordinaires et les supérieurs les pourront contraindre comme rebelles et incorrigibles ". Le paradoxe est évident : dans une ère où l'effort catholique trouve en la femme une alliée toute dévouée, celle-ci, si elle se veut compter aux rangs de l'Église, se voit interdire le mouvement essentiel à toute activité missionnaire. La femme dont la vocation serait à la fois religieuse et apostolique fait donc face à un dilemme : le choix de la religion implique l'abandon de tout engagement dans le monde, tandis que le choix de l'action rend tout statut religieux impossible.

\section{Religieuses et actives : des congrégations calquées sur les clercs réguliers}

À travers l'Europe du XVII ${ }^{\mathrm{e}}$ siècle, l'impulsion apostolique féminine se fait sentir de façon très nette : de nombreuses communautés se forment spontanément sur le modèle évangélique de la vie mixte, plus proche de la vie du Christ que celui proposé par les moniales traditionnelles. Or, malgré leur ardent désir d'être approuvées par le Saint Siège, ces congrégations ne bénéficient alors d'aucun statut religieux officiel puisqu'elles ne se conforment pas aux règles monastiques mais au contraire délaissent la sédentarité et la séparation qui vont de pair avec le couvent pour se dévouer à un apostolat demandant une interaction quotidienne avec le peuple. Ainsi réunies dans un but pieux, ces femmes qui se veulent faire partie de l'Église en

\footnotetext{
${ }^{5}$. Cité dans Jean-Baptiste THIERS, Traité de la clôture ..., op. cit., p. 68.
} 
enfreignent donc pourtant les règles ; leur histoire est scandée des difficultés liées à leur décision de ne pas se cloîtrer.

Parmi ces nouvelles communautés actives à but religieux, les Ursulines sont le fer de lance de la mission pédagogique et catéchétique française. Si la recherche fait en général honneur à la congrégation de Paris, c'est pourtant bien la fondation de Toulouse, fort méconnue, qui en 1604 lance l'initiative ursuline en France hors de la Provence et du Comtat Venaissin. Simples congrégées dénuées de statut officiel, elles sont prêtes à braver l'opprobre pour se vouer à une vocation qu'elles estiment plus sainte même que la vie monastique traditionnelle. L'apostolat se trouve en effet au cœur de la démarche ursuline et les jeunes femmes notent que c'est leur mode de vie, si proche de celui du Christ, qui donne à leur action toute sa valeur :

“La vie des Ursulines n'est autre que celle que le fils de Dieu a menée en ce monde, et sa Sainte mère avec les apôtres après sa montée au ciel. Voilà les modèles et les exemplaires de leur état et profession: c'est la vie mixte mêlée de la contemplation et de l'action ... la voie la plus assurée et la plus profitable à la gloire de Dieu, au bien de son Église et au salut des âmes”.

L'imitation du Christ semble ratifier une vocation religieuse non cloîtrée ; c'est elle qui justifie et motive à la fois l'entreprise des premières Ursulines qui se perçoivent comme des "anges tutélaires” envoyées par Dieu afin d'assurer le salut des âmes des jeunes élèves et des femmes qu'elles instruisent. Leur sexe, loin d'être incompatible avec une telle vocation apostolique, fait au contraire partie du dessein divin qui utilise les instruments les plus fragiles et les plus humbles pour réaliser son œuvre. Selon elles, Dieu "se sert de ces chétifs instruments pour faire la guerre à l'enfer, pour détruire le vice, établir le règne des vertus et conserver l'innocence dans les âmes" . La femme a donc sa place au sein des efforts de reconquête du peuple entrepris par les mouvement missionnaires de la contre-réforme ; la congrégée se veut être l'instrument de Dieu et l'alliée de l'Église.

\footnotetext{
${ }^{6}$. Archives départementales de la Haute-Garonne (désormais AD31), 221H-37, f. 2, Mémoires du commencement et progrès de l'ordre de Sainte Ursule, Cahier 1604-1621.
} 
La vigueur de ces vocations n'est en aucun cas limitée au continent et on constate qu'Outre-Manche, bien des fidèles s'investissent aussi corps et âme dans la lutte contre le protestantisme établi. Si un nombre croissant de dévotes n'hésite plus à s'expatrier pour entrer dans les Ordres, la survie de la communauté récusante et le second souffle vital qu'elle trouve sous le règne de Jacques I doivent beaucoup aux efforts de celles qui, restées en Angleterre, se résignent à demeurer laïques faute de couvents sur leur sol natal. À ces deux solutions traditionnelles - aut maritus, aut murus, l'enfermement domestique ou celui du cloître - l'Institut des Dames anglaises qui voit le jour à Saint Omer en 1611 vient ajouter une alternative sans précédent. C'est la première fondation anglaise à s'éloigner de la tradition cloîtrée : à l'instar des Ursulines françaises, les disciples de la fondatrice Mary Ward se targuent d'imiter les clercs réguliers et de servir l'Église tout en demeurant dans le siècle.

En 1616, alors qu'elles tentent de gagner l'approbation papale, elles rédigent le Ratio Instituti, document qui explique la raison d'être de leur Institut :

"Puisque 1'Angleterre dans son grand malheur a tant besoin de travailleurs spirituels et puisque les prêtres, apôtres de cette récolte, s'y emploient si assidûment, il semble que face à cette même nécessité spirituelle, le sexe féminin doive et puisse lui aussi selon sa propre mesure apporter une contribution comparable"7.

Le lien causal qui s'établit ici entre le besoin de missionnaires en Angleterre et l'entreprise de Mary Ward traduit avec simplicité l'esprit pragmatique de l'Institut. Loin de refuser le cloître dans un mouvement de rébellion contre les canons de l'Église, c'est dans un esprit de service que les Dames anglaises choisissent l'action. Comme les Ursulines, elles ne voient pas d'incompatibilité entre leur sexe et leur vocation, mais plutôt une réponse fonctionnelle à un besoin concret car, selon la fondatrice, si les garçons bénéficient déjà de l'aide des prêtres missionnaires, les filles, tout aussi nombreuses, sont laissées pour compte. Les Dames anglaises se

\footnotetext{
${ }^{7}$ Archives du Bar Convent, York (désormais ABC), B18, Ratio Instituti, f. 1. Fondé clandestinement par les Dames anglaises en 1686, le Bar Convent est encore en activité à ce jour et a l'honneur d'être le plus ancien couvent du royaume. L'Institut de la Bienheureuse Vierge Marie y conserve une partie de ses archives.
} 
proposent donc de compléter le mouvement masculin en catéchisant l'autre moitié de la population, assurant ainsi la continuation de la foi chez les futures mères de famille ${ }^{8}$. Ainsi, elles s'inscrivent au sein du mouvement de la contre-réforme et calquent leur formation sur le modèle de la mission jésuite alors si ardente ; elles quittent d'abord leur pays pour être formées sur le continent avant de s'appliquer à instruire les populations d'Europe et surtout d'Angleterre, où beaucoup retournent clandestinement ${ }^{9}$.

Initialement, Ursulines françaises et Dames anglaises adoptent un fonctionnement similaire. Libres de leurs mouvements, elles ouvrent des écoles où internes et externes apprennent à lire et à écrire tout en acquérant les rudiments de la doctrine catholique. Dès le début, elles se déplacent non seulement de ville en ville mais aussi (surtout dans le cas de l'Institut) de pays en pays, fondant partout de nouveaux établissements. Durant leurs premières années, ces nouvelles religieuses apostoliques présentent pour la plupart un profil qui n'a a priori rien de menaçant ni pour l'Église ni pour la société patriarcale dans laquelle elles évoluent: œuvres charitables, visite des malades et des prisonniers et enseignement des filles sont en effet depuis toujours des activités féminines. Cependant, Ursulines françaises et Dames anglaises ne se limitent pas à ces tâches philanthropiques et domestiques ; elles entreprennent non seulement de sauvegarder la foi là où elle est présente mais aussi de convertir des femmes de mauvaise vie, des non croyantes et des huguenotes voire, à l'occasion, des hommes. Cette mission n'a alors plus rien de féminin et ressemble de très près à celle des prêtres et des clercs séculiers.

Le nouveau modèle de la religieuse à vocation apostolique soulève des problèmes d'ordre déontologique autant que constitutionnel. Par son défi de la

\footnotetext{
8. ABC, B18, Brevis Declaratio.

${ }^{9}$. La fondation créée par Mary Ward ne recevra jamais de nom officiel et nous la désignerons ici sous le nom de l'Institut des Dames anglaises. Cette première phase de l'Institut prend officiellement fin en 1631, quand Urbain VIII ordonne sa suppression à l'issue d'années de controverse. Cependant, la fondation renaîtra de ses cendres et finira par être approuvée par Clément XI en 1702. Cette seconde phase connaîtra plus de succès que la première, puisque le nouvel Institut, nommé Institut de la Bienheureuse Vierge Marie, est aujourd'hui implanté dans le monde entier.
} 
tradition, celle-ci fait preuve d'un esprit d'indépendance qui ne peut que mettre en péril l'ordre établi. C'est donc à cause de sa vocation prosélyte qu'elle se met à dos tant de détracteurs, dont les deux griefs essentiels - l'un lié aux préconceptions misogynes du siècle, l'autre centré sur des considérations canoniques - se concentrent sur la liberté de mouvement et l'interaction avec le monde. En tête de la liste de doléances dressée par le clergé en réponse à la vague d'innovation qu'il s'efforce de contenir, les déplacements excessifs sont jugés nuisibles aux mœurs des femmes, qu'elles soient d'Église ou pas; ils sont également incompatibles avec le statut religieux féminin, qui exige la soumission à la clôture. Le préjudice est donc double, condamnant les Ursulines françaises et les Dames anglaises à cause de leur sexe mais aussi de leurs aspirations religieuses qui menacent les privilèges masculins.

L'opposition suscitée par leur manque de clôture met très vite en péril l'existence même de ces congrégations qui choisissent alors des voies opposées : afin d'éviter la suppression les Ursulines françaises acceptent le voile, mais sous certaines conditions. La “congrégation féminine enseignante par excellence” inaugure ainsi une nouvelle formule ouvrant pour la première fois les classes du cloître à des élèves externes, entreprise couronnée d'un tel succès qu'on compte en France un total de 320 communautés avant la fin du siècle ${ }^{10}$. Face aux mêmes difficultés les Dames anglaises, elles, refusent de compromettre leur liberté de mouvement ; malgré la controverse et la multitude de difficultés qu'une telle décision engendre, leur vocation religieuse se poursuit donc au sein de la société séculière, loin des restrictions du couvent.

\section{Laïques, indépendantes et sans statut : les femmes que l’on méprise}

Toute analyse des difficultés liées au déplacement des femmes à vocation religieuse se doit de considérer la nature duelle des arguments invoqués par les

\footnotetext{
${ }^{10}$. E. RaPley, The Dévotes..., op.cit., p. 48 ; estimation de M.C. Gueudré, Histoire de l'ordre des Ursulines, 2 vols, Paris, St Paul, 1960, ii, p. 148.
} 
détracteurs des congrégations non cloîtrées. Avant même d'en venir aux considérations de statut religieux, l'opposition naît d'abord du fait qu'il s'agit du sexe dit faible. La femme, selon le trope bien connu de l'époque moderne, est non seulement plus fragile, plus vulnérable mais aussi plus susceptible d'être séduite, sa vertu et son intellect étant inférieurs à ceux de $1^{\prime}$ homme ${ }^{11}$. La mobilité géographique au féminin n'est pas sans évoquer une certaine inconstance morale, ni sans remettre en question honnêteté et humilité. La liberté du corps symbolise le vagabondage des mœurs, l'indocilité et la perversion. Le scandale enveloppe la voyageuse tel un manteau de murmures et d'accusations.

Cause, ou peut-être symptôme d'une absence de stabilité intérieure et d'un manque des jalons moraux essentiels pour ne point s'éloigner des sentiers battus, la pérégrination est associée au vice et à l'inconduite. Une femme sillonnant les routes ne peut que susciter la méfiance. C'est donc sans grande surprise que l'on constate que quand deux Ursulines d'Avignon, Marguerite de Vigier et Françoise de Blanchet, partent fonder une communauté à Toulouse en 1604, elles sont appréhendées par les autorités municipales de la ville de Pézenas et jetées en prison, soupçonnées d'être des femmes de mauvaise vie ${ }^{12}$. Cet incident n'est pas un cas isolé, l'histoire des Ursulines de France est jalonnée de tels témoignages d'hostilité. En 1606, Geneviève de Valembert et Christine Peiron fondent un établissement dans la ville de Grenoble et sont bientôt en proie à l'opprobre : “Un fameux prédicateur déclama contre elles en pleine chaire dans l'église de sainte Claire, et les montrant du doigt et les nommant par leur nom, dit quantité de choses qui leur étaient très désavantageuses "13. Si, dans ce cas, les attaques proviennent d'un clerc, il n'est pas rare qu'elles émanent du public et très souvent même des enfants. À Dijon, le peuple tourne le dos à Françoise de

\footnotetext{
11. Voir entre autres Lucy De BRUYn, Woman and the Devil in Sixteenth Century Literature, Tisbury, Compton Press, 1979 ; C.F. KARLSEN, The Devil in the Shape of a Woman, New York, W. W. Worton, 1987 ; Ian MACLEAN, The Renaissance Notion of Woman, Cambridge, Cambridge University Press, 1980 ; Margaret SommerviLLE, Sex and Subjection: Attitudes to Women in Early Modern Society, Londres et New York, Arnold, 1995 et Alison Weber, 'Little Women : Counter-Reformation Misogyny', in David LuebKe (dir.) The Counter-Reformation. The Essential Readings, Oxford, Blackwell, 1999, p. 143-62.

12. AD31, 221H-37, Mémoires du commencement et progrès de l'ordre de Sainte Ursule, f. 29.

13. Mère de Pommereu, Ursuline (désormais MDPU), Les Chroniques de l'ordre des Ursulines recueillies pour l'usage des religieuses du mesme ordre, Paris, Eloy Hélie, 1676, i, p. 84.
} 
Xainctonge, fondatrice de la nouvelle congrégation d'Ursulines. Ayant quitté sa famille, l'étiquette voudrait qu'elle prenne époux ou qu'elle se cloître et non qu'elle aille de part et d'autre de la ville flanquée de ses compagnes ${ }^{14}$. Ce sont les enfants qui verbalisent l'anxiété et la peur des adultes à force d'insultes, de chansons humiliantes, voire de crachats et de jets d'immondices. Les congrégées sont exclues de la société ; craintes par les uns et ridiculisées par les autres, elles symbolisent le refus des principes patriarcaux qui régulent le siècle et, de ce fait, elles menacent la stabilité $\mathrm{du}$ système. Conscientes des difficultés liées à leur indépendance et à leur manque de statut officiel, les Ursulines de France décident donc, dans la première moitié du $\mathrm{XVII}^{\mathrm{e}}$ siècle, d'abandonner leur liberté de mouvement et de prendre le voile, avec le vœu de clôture perpétuelle qu'il implique.

Face à des difficultés similaires l'Anglaise Mary Ward, elle, rejette le cloître et refuse de soumettre son Institut à une autorité épiscopale qu'elle estime mal à propos. D'après les conceptions d'ancien régime, qui confinent la femme soit au domaine $\mathrm{du}$ foyer soit au couvent, l'entreprise de Mary Ward ne peut que paraître équivoque et les calomnies sont omniprésentes dans l'histoire de celles qu'on affuble de sobriquets tels que " nonnes errantes" (“wandering nuns"), "galopeuses" (" galloping girls") ou “commères errantes" (“wandering gossips"), que l'on suspecte en outre de relations immorales avec les Jésuites ${ }^{15}$. La pétition adressée à Grégoire XV par le frère bénédictin Robert Sherwood (1588-1665), adversaire de la Société de Jésus, dénonce les innovations de l'Institut et abonde de sous-entendus remettant en question la chasteté de la fondatrice et de ses consœurs - et plus particulièrement l'honnêteté de leurs rapports avec les pères jésuites qu'elles côtoient ${ }^{16}$.

$\mathrm{Au}$ cœur de la tourmente, Mary Alcock, ancienne sœur de l'Institut, se retourne contre lui pour dénoncer son mode de vie ; son témoignage fournit aux

\footnotetext{
${ }^{14}$. Ibidem, part 4, p. 3-9.

${ }^{15}$. Lewis Owen, Running Register, Londres, Milbourne, 1626.

${ }^{16}$. ABC, B 45, Joseph GRISAR, The First Accusations in Rome against the Institute of Mary Ward (1622), p.28. Traduit de l'allemand par M. CAMPION DAVENPORT.
} 
détracteurs de l'Institut les armes nécessaires pour dénoncer le scandale qu'il représente. Dans une attaque virulente, Alcock raconte comment sous prétexte de préserver leur anonymat et de cacher la nature religieuse de leurs activités dans le centre clandestin d'Hungerford House, à Londres, les Dames anglaises s'habillent de soie, de velours et de collerettes de couleurs vives, attirent l'attention de tous par leur train de vie luxueux et sont régulièrement prises pour des courtisanes et des prostituées ${ }^{17}$. L'une d'entre elles est même accusée d'attendre un enfant. Ainsi les Dames anglaises font-elles l'objet de la méfiance des "Catholiques de bonne conscience " selon qui, au dire de Thomas Fuller " ces dames errantes dévient ... de la modestie féminine (pour ne pas dire virginale)". Et d'ajouter, non sans un certain sarcasme, que "si les hommes ne font que circuler, les femmes, elles, vagabondent $" 18$.

Le monde voit dans la mobilité des Dames anglaises toutes les indications d'un tempérament inconstant. Outre la corruption de la vertu, le mouvement va de pair avec un manque d'humilité qui entache la fondatrice, ses consœurs et même leurs élèves. Celles-ci contreviennent tant à la discrétion et à la docilité qu'elles sont, dans l'esprit de leurs critiques, des femmes-hommes, usurpant jusqu'à l'identité masculine ; Mary Ward est d'ailleurs accusée de vivre au sein d'un collège de jésuites déguisée en habit de prêtre. Chez les religieuses missionnaires, la mobilité physique semble indiquer une nature plus masculine que féminine. En 1630, alors que 1'Institut fait l'objet d'une enquête ordonnée par le saint siège, le secrétaire de Propaganda Fide, Francesco Ingoli, s'insurge contre l'obstination de la fondatrice à transgresser les rôles de son sexe. Il décrit Mary Ward comme " une vierge à l'esprit viril ", faisant ainsi écho à une de ses déclarations antérieures où elle lui semble être une "jeune femme au tempérament masculin $"$. Au cours de son investigation, Ingoli recueille de nombreux témoignages afin de déterminer la nature de l'Institut et d'en juger le

\footnotetext{
${ }^{17}$. ABC, C1, Godfather's Information about the Jesuitesses (1623).

${ }^{18}$. Thomas FuLLER, The Church History of Britain from the birth of Jesus Christ, until the year 1648, Londres, J. Williams, 1655, vi, p. 498.

19. ABC, C18, f. 101, Francesco Ingoli, 1627.
} 
bien-fondé. Il interroge sept Dames anglaises qui toutes restent fidèles à Mary Ward et à l'idéal missionnaire de leur communauté ; mais c'est surtout la masse des dépositions outrées par l'affront moral que représente un groupe de femmes non cloîtrées qui le pousse, dans le Compendium qu'il soumet au pape en conclusion de son rapport, à presser le pontife de dissoudre l'Institut sans tarder ${ }^{20}$.

\section{Des religieuses à vocation apostolique : la vie mixte au féminin}

La virulence de l'opposition qui se dresse contre l'Institut des Dames anglaises peut surprendre. Il faut se tourner vers les activités de ces congrégations pour être à même d'interpréter les réactions qu'elles suscitent: au contraire des Ursulines, dont la mobilité initiale est remplacée par la clôture au cours du XVII siècle, les Dames anglaises sont décidées à imiter les jésuites autant que possible et concrétisent leur idéal missionnaire en refusant le couvent. Leur tâche est double puisqu'il s'agit à la fois de maintenir une présence constante chez les catholiques clandestins d'Angleterre et de convertir les protestants. La plus ancienne biographie de Mary Ward explique comment elle travaille incognito dans les rues de Londres parmi les infortunés qu'elle rencontre chaque jour ${ }^{21}$. Quand en 1639, après de longues années d'exil, elle est de retour à Londres, elle ouvre un centre permettant à la communauté récusante de la capitale d'y venir prier, se confesser et entendre la messe dite par l'un des deux prêtres qui y résident en permanence. Malgré les dangers de la répression protestante, la fondatrice inaugure une école primaire qui se voit confier l'éducation des filles des familles catholiques les plus influentes ainsi que celle des plus humbles.

Les activités des membres de l'Institut font écho à celles de leur supérieure : le rapport écrit au début des années 1620 par une missionnaire clandestine nommée sœur Dorothea met en lumière la nature de ses activités pédagogiques et évangéliques. À la

\footnotetext{
${ }^{20}$. ABC, C1, Letters against the Jesuitesses, ff. 309-312. Il interroge Maria Clopey, Elizabeth Hall, Anna Morgan, Catherine Smith, Brigida Heyd, Winefrid Campion and Anna Micel.

${ }^{21}$. ABC, B12, A Briefe Relation of the Holy Life and Happy Death of our Dearest Mother, écrite à titre posthume par ses plus proches compagnes Mary Poyntz et Winefrid Wigmore c. 1650, f. 20.
} 
tête d'un petit centre sous la protection de Lady Timperley de Hintlesham Hall, dans le Suffolk, Dorothea voyage de famille en famille, éduque les enfants, tente de consolider la foi des parents et s'efforce d'atteindre les pauvres ("the simple and vulgar sort ") ${ }^{22}$. Dans ce journal de bord, les silences sont tout aussi éloquents que les mots : on comprend donc que, par souci de sécurité, la jeune femme ne révèle nulle part sa véritable identité civile. Mais ses priorités sont également inscrites dans le texte et si elle reste très vague sur le contenu et les méthodes de ses activités d'institutrice, elle décrit ses travaux de prosélyte dans de plus amples détails. On constate donc à travers ce témoignage que, si essentielle que soit l'aide apportée aux catholiques, les Dames anglaises aspirent à dépasser ces activités pastorales pour amener les protestants à se convertir. La lecture du rapport de Dorothea démontre que l'interaction avec le peuple et la liberté de mouvement sont deux des piliers essentiels de la mission des Dames anglaises : elle doit être libre d'aller au-devant des sujets qu'elle désire rencontrer et de couvrir de longues distances pour entrer en contact avec le prêtre le plus proche. Ainsi, alors même qu'elle se réjouit de la conversion de neuf personnes, la missionnaire doit parcourir près de quinze kilomètres à pied pour solliciter un jésuite ${ }^{23}$. Il lui est essentiel de pouvoir se déplacer : sans mouvement, il n'est point de mission pour les Dames anglaises.

Si le désir d'explorer de nouveaux territoires est caractéristique du XVII ${ }^{\mathrm{e}}$ siècle en général et de l'engouement naissant pour le voyage, il n'inclue d'habitude que très peu de femmes. Or, Mary Ward va sillonner toute l'Europe, souvent à pied ou à dos d'âne; sa motivation première n'est pas cependant la découverte de l'inconnu mais le désir de propager son Institut. Elle entretient tout au long de sa vie des liens privilégiés avec sa terre natale, dont la conversion est la raison d'être de cette Société de Jésus au féminin. Ainsi, elle traverse la Manche plus de dix fois, dans des conditions hasardeuses, pour revenir servir ses compatriotes ${ }^{24}$. Sur le sol anglais,

\footnotetext{
${ }^{22}$. M.C.E. CHAMBERs, The Life of Mary Ward, 1585-1645, 2 vols, Londres, Burns and Oates, 1882-1885, ii, p. 27 39.

23. Ibidem, p. 28.

${ }^{24}$. Ses premières compagnes sont Mary Pointz, Winefrid Wigmore, Johana Browne, Susanna Rookwood et Katherine Smith. Barbara Babthorpe et Barbara Ward sont supposées avoir également fait partie du voyage. Voir
} 
elle réside à diverses reprises dans la capitale, visite Canterbury pour finir son périple dans son Yorkshire natal. En route, elle passe quelques mois à Newby et Ripley Castle, puis atteint la petite communauté d'Hutton Rudby en septembre 1642; en 1643, accompagnée de quelques fidèles, elle ouvre un nouveau centre récusant dans le village de Heworth, aux portes de York, avant d'y mourir deux années plus tard, en janvier $1645^{25}$

Une telle mobilité est en elle-même remarquable pour toute femme de l'époque moderne, mais les pérégrinations de la fondatrice ne se limitent pas à l'Angleterre. Sur le continent, elle fonde de nombreuses branches de son Institut : après Saint Omer en 1611, c'est le tour de Liège en 1616, de Cologne et de Trèves en 1620-1, de Rome en 1622, de Naples et de Pérousse en 1623, de Munich et de Vienne en 1627 et enfin de Pressburg et de Prague en 1628. Ses voyages l'amènent donc aux quatre coins de l'Europe, poussant au Nord jusqu'à la ville de York, au Sud jusqu'à Rome (où elle se rend trois fois) et à l'Est jusqu'à Prague, aux portes occidentales de l'empire ottoman. Elle s'arrête en chemin dans de diverses villes comme Lorette, Assise, Venise, ou $\mathrm{Spa}^{26}$. Son dernier périple, au cours duquel elle manque de perdre la vie, couvre les milliers de kilomètres qui séparent Rome du Yorkshire, négocie les cols périlleux des Alpes en plein hiver et fait halte tour à tour dans des villes comme Cologne ou Paris. Un tel parcours ne peut qu'être exceptionnel pour son siècle et n'est pas sans susciter l'hostilité au sein même de l'Église à laquelle Mary Ward se proclame si dévouée.

L’Institut apparaît donc à ses contemporains comme défini par sa mobilité même, et en termes toujours négatifs : on reproche à celles que l'on surnomme les “nonnes errantes" leur imitation des pères jésuites et de leurs activités " de par le

Henriette Peters, Mary Ward: A World in Contemplation, traduit en anglais par Helen ButTerworth, Leominster, Gracewing Books, 1994, p. 110.

${ }^{25}$. Après la mort de la fondatrice, les Dames anglaises n'opèrent plus en Angleterre, mais elles sont de retour en 1669 avec une fondation à Hammersmith, suivie d'une seconde à Hammersmith en 1678 et enfin d'une troisième à York en 1686. Le couvent de York continue d'être actif de nos jours.

${ }^{26}$. L'extraordinaire mobilité physique de Mary Ward est l'objet d'une analyse par Joseph GrISAR, spécialiste de l'Institut, dans 'Mary Ward, 1585-1645', The Month, 12.22, 1954, p. 69-82. 
monde, prêchant l'évangile à celles de leur sexe en Angleterre et ailleurs " ${ }^{\text {27 }}$. Cette constante comparaison avec la société d'Ignace de Loyola est revendiquée par Mary Ward elle-même : en 1621, la fondatrice cherche à obtenir l'approbation papale et adresse à Rome une requête dans laquelle elle décrit le fonctionnent de sa nouvelle “ Société de Jésus ". Dans ce texte, connu sous le nom d'Institutum, elle sollicite l'envoi de ses consœurs partout dans le monde au même titre que les jésuites. Les “ jésuitesses" y expriment le désir d'être mandatées directement par le pape pour aller travailler "parmi les Turcs ou tout autre peuple infidèle, même chez ceux qui vivent dans la région qu'on nomme les Indes, ou parmi les hérétiques de toute sorte ${ }^{{ }^{2} 8}$. La mission des Dames anglaises méprise les frontières, et elle reste en cela une entreprise unique.

Cette mobilité d'un même groupe à l'échelle internationale n'est partagée, à notre connaissance, par aucun autre groupe d'aspirantes religieuses. Pour ce qui concerne les Ursulines françaises, un tel déploiement spontané devient impossible dès lors que les simples congrégations laïques des débuts se transforment progressivement en cloîtres au cours du XVII ${ }^{\mathrm{e}}$ siècle ; le rayonnement géographique des Ursulines reste donc plus modeste, en général à l'échelle locale. En outre, ce n'est qu'avec licence épiscopale que les maisons peuvent envoyer certaines de leur sœurs fonder de nouveaux établissements, qui eux aussi seront clos. Les évêques ont d'ailleurs grand soin d'exhorter ces fondatrices à la plus grande diligence et à la plus grande retenue lors du voyage nécessaire: " nous vous avons permis et permettons par ces présentes ... de revenir au collège de Ste Ursule de cette ville ... sans vous divertir ailleurs et avec la décence et modestie convenable a votre profession "29.

C'est ce cette façon que les Ursulines de Toulouse s'établissent entre autres à Brives-la-Gaillarde en 1608, à Limoges en 1620, à Bayonne en 1621, à Auch en 1623, à Villefranche de Rouergue et à Grenade en 1626, à Béziers en 1632 et à Pamiers en

\footnotetext{
27. James WADSWORTH, English Spanish Pilgrim, Londres, Michael Sparke, 1629, p. 30.

${ }^{28}$. ABC, B18, Institutum, f. 22.

${ }^{29} \mathrm{AD} 31,221 \mathrm{H}-15$, f. 78 . Licence datée du 13 janvier 1651 pour le retour d'une Ursuline toulousaine dans son couvent après la fondation d'une nouvelle maison à Pamiers.
} 
1644. En parallèle, d'autres branches comme celles de Paris ou de Bordeaux essaiment à leur tour, assurant ainsi rapidement la présence ursuline sur tout le territoire. Mais si la pratique leur interdit la mobilité physique, les Ursulines ne sont pas cependant sans s'intéresser aux régions lointaines auxquelles Mary Ward aspire. Compte tenu de la sévérité des règles qui contrôlent les lectures des sœurs, tant dans leur correspondance privée que dans leurs études, il n'est pas insignifiant de noter qu'elles conservent dans leurs archives des récits comme la Relation de la conquête du Mogol par Thamas-Koulikan, et de la façon de vivre des dames mahométanes envoyée par un missionnaire au Bengale à la sœur Hyacinthe de Toulouse ${ }^{30}$.

L'idéal de conversion universelle est présent chez les Ursulines comme chez les Dames anglaises. Catherine Canterel, de la communauté d'Amiens, entretient des liens particuliers avec les pères jésuites et, bien qu'elle ne puisse elle-même se joindre à la mission catholique en cours au Canada, développe une relation très forte avec les pères Brebœuf, Le Jeune, Ragenau et de Caen, missionnaires chargés de cette ambitieuse entreprise ${ }^{31}$. Loin d'être exceptionnelle dans sa conception mondiale de l'engagement des Ursulines, Catherine Canterel représente nombre de ses consœurs, comme en témoigne l'empressement de ces religieuses pour la mission canadienne. À Lisieux, la notice mortuaire de Marie Godebit, avide lectrice de dépêches provenant du Canada, commente : "Son zèle pour le salut des âmes la portait à offrir ses prières pour la conversion du Canada, demandant instamment à Dieu qu'il donna force et efficace aux paroles des personnes qui travaillent au salut de ces pauvres peuples ${ }{ }^{2}$. À Evreux, Elizabeth Turgis se porte volontaire pour l'exil en Nouvelle France, mais la mission ne peut alors accueillir d'autres religieuses. Déçue, elle ne perd rien de son enthousiasme et elle prend la tête de l'externat de sa communauté, qu'elle désigne comme " son cher petit Canada ”. Dans l'attente de voir un jour ce nouveau pays, elle

\footnotetext{
30. Relation de la conquête du Mogol par Thamas-Koulikan, et de la façon de vivre des dames Mahométanes ; écrite par un missionnaire français qui est dans le royaume de Bengale, à Mad. de S. Hyacinthe, religieuse Ursuline à Toulouse (Toulouse, 1740).

31. MDPU, Les Chroniques ..., op.cit., iii, p. 421.

${ }^{32}$. Bibliothèque de l'Arsenal, Paris (désormais BA), ms 4990-56 - Lisieux, 2 mai 1686.
} 
surnomme ses externes ses "petits sauvageons" "33. Bien d'autres comme elle demandent à être envoyées à leur tour sur la terre des "sauvages ", si bien que le Jésuite supérieur du Québec s'étonne du nombre d'Ursulines souhaitant le rejoindre outre-Atlantique ${ }^{34}$.

L'Ordre est de nos jours célèbre pour son implantation précoce dans les régions hostiles du Canada, sous la direction de Marie Guyart (1599-1672), en religion Marie de l'Incarnation, fondatrice du mouvement des Ursulines au Québec ${ }^{35}$. À son arrivée en 1639, aidée de deux compagnes, elle se voit confier l'éducation séculière et religieuse de six jeunes natives; fidèle aux pratiques des prosélytes de l'époque, elle utilise le dialecte local et apprend l'Algonkin, le Huron et l'Iroquois pour mieux communiquer son message au peuple. Les difficiles conditions de vie font que ses activités sont très similaires à celles des pères jésuites. Les religieuses missionnaires ne peuvent observer les règles de la clôture et doivent se déplacer fréquemment, ne serait-ce que pour se procurer des vivres et des matériaux combustibles. Les circonstances permettent alors aux Ursulines d'adopter le modèle évangélique de la vie mixte d'une façon qui leur est interdite sur leur terre natale ${ }^{36}$. Cependant, une telle mobilité physique reste exceptionnelle et la plupart des postulantes se verront refuser leur vœux: le Québec est en effet le théâtre d'affrontements violents entre colonisateurs et indigènes et l'Église ne peut y approuver la présence de religieuses dont le manque de clôture met en péril leur vertu et jusqu'à leur vie. Ainsi, les réussites de Marie de l'Incarnation demeurent des exploits auxquels la majorité de ses consœurs ne peut qu'aspirer.

LES OBJECTIONS CANONIQUES : LE MOUVEMENT, SYNONYME D'USURPATION

\footnotetext{
${ }^{33}$. MDPU, Les Chroniques..., op.cit., iii, p. 288-90.

${ }^{34}$. Reuben Gold Thwaites (dir.), The Jesuit Relations and Allied Documents, 73 vols, Cleveland, Ohio, Burrows, 1896-1901, vol. 6, p. 142.

35. Dom Guy Marie OuRY, Marie de l'Incarnation 1599-1672, Tours, Abbaye Saint Pierre de Solesmes, 1973.

36. Charles SAINTE-FoI, Vies des premières Ursulines de France tirées des chroniques de l'ordre, Paris, Poussielgue-Russand, 1856, p.163-222.
} 
Les exemples fournis par les Ursulines françaises et les Dames anglaises illustrent l'un des phénomènes principaux de la réforme catholique, cet engouement féminin marqué notamment par la multiplication de compagnies séculières noncloîtrées. Cependant l'Église, même en pleine mission de reconquête du peuple, n'est pas prête à accepter la liberté de mouvement pour les congrégations féminines. Alors qu'en France comme en Angleterre la mission jésuite connaît un succès remarquable, cette victoire demeure sans contrepartie pour les femmes. L'opposition au mouvement des moniales est complexe, liée tant aux objections misogynes dévoilées plus haut qu'à l'aspiration de ces femmes à un statut religieux, qui implique la sujétion à l'autorité de l'Église et un rôle à la définition aussi étroite qu'elle est immuable.

Or, bien que cloîtrées, les Ursulines s'efforcent d'honorer leur vocation apostolique et entretiennent des liens privilégiés avec le monde ; pour nombre d'entre elles, le couvent ne doit pas être synonyme d'isolement complet. La toulousaine Claire Durdes incarne ce désir d'action : l'archevêque Charles de Montchal l'envoie pour un temps à la maison de la Charité en qualité de supérieure. Là, elle instruit les femmes débauchées en voie de réhabilitation et en convertit huit qui deviennent religieuses. Enfin, elle est mandatée plus loin encore, pour fonder les Ursulines de Pamiers " pour le bien et le salut des âmes ". Compensant les restrictions de la clôture par la dispersion la plus large possible de son catéchisme, elle fait de la fondation de Pamiers un emblème de zèle et d'excellence. Elle doit bientôt ouvrir une deuxième classe pour les externes et les femmes de la ville se querellent pour assister à ses leçons dominicales de doctrine dont le succès est tel que les hommes viennent se joindre à l'audience. Claire Durdes fait tout pour attirer les non-Catholiques à ces leçons et ses pouvoirs de persuasion doivent être remarquables, car Parayre note la conversion de quatre huguenotes, qui deviennent elles-mêmes Ursulines. Elle reçoit chaque dimanche plus de quatre-vingts personnes à la grille de l'église, et les 
témoignages contemporains indiquent qu'on préfère venir l'écouter plutôt que le père de Saint Denis, envoyé par l'évêque pour faire la doctrine ${ }^{37}$.

Claire Durdes brouille dangereusement les limites qui séparent l'enseignement privé et la prédication. Si elle prêche publiquement, n'usurpe-t-elle pas le rôle des prêtres tels que le père Saint Denis ? Pour reconvertir une région fortement influencée par le protestantisme, la jeune pédagogue s'octroie un rôle militant qui, s'il sied aux clercs réguliers, n'est pas traditionnellement du ressort des religieuses. Elle contrevient aux injonctions de saint Paul (I Tim. 2:12) et aux décrets tridentins qui interdisent à la femme de prêcher en public. Consciente du danger et avec une prudence toute ursuline, Durdes se hâte donc d'obtenir une licence l'autorisant à instruire les adultes dans le chœur bas de l'église, de façon à poursuivre ses activités en toute sécurité.

Après l'expiration de son mandat à Pamiers, elle rentre à Toulouse dans les années 1650 et prend la charge de mère de la Compagnie des Dames de la Miséricorde de Sainte Ursule. L'existence même de cette Compagnie témoigne du désir que les Ursulines ont de garder un lien avec le monde : formé alors que la congrégation de Toulouse est transformée en couvent en 1616, ce groupe constitué de vierges, de mariées et de veuves dévotes sert de bras droit au nouveau couvent, continuant au sein de la ville les travaux apostoliques que les Ursulines doivent abandonner. Depuis le parloir, cette zone intermédiaire entre les mondes de l'intérieur et de l'extérieur, Claire Durdes supervise les activités des Dames de la Congrégation : bien que physiquement isolée, elle possède une influence qui infiltre le monde séculier au quotidien, et grâce à sa relation avec la compagnie l'action ursuline se fait sentir au delà des murs du couvent. Durdes est également employée aux classes, où elle trouve l'occasion de dépasser l'hermétisme de l'enfermement monastique : si elle ne sort plus du monastère, les externes viennent y puiser son message évangélique pour à leur tour le disséminer dans le monde. 37. . P. PARAYRE, Chronique des religieuses augustines ursulines de la congrégation de Toulouse, 3 vols,
Toulouse, Guillaume Bosc, 1681, iii, p. 2-21. 
L'exemple de Claire Durdes illustre la complexité de la relation entre clôture et liberté. En effet, si la clôture peut certes être prohibitive, elle n'en permet pas moins la propagation de l'apostolat pédagogique des Ursulines; bien qu'elle ne travaille jamais qu'à l'intérieur de la clôture, l'Ursuline n'est pas entièrement recluse et sa claustration corporelle ne nuit pas à la dissémination de son catéchisme ${ }^{38}$.

Cependant, c'est le succès même de la diffusion du message des Ursulines qui, paradoxalement, peut mettre ces dernières en danger. En effet, l'une des préoccupations majeures reste l'appropriation des rôles traditionnellement masculins par des femmes missionnaires. Les hommes sont par essence habilités à être en contact avec le peuple, à prêcher en public. Mais les nouvelles religieuses à vocation active s'octroient et usurpent ces mêmes attributs. Il y a donc là une rivalité implicite. L'exemple de Françoise Rabonite, dite sœur de Sainte Claire, illustre parfaitement l'importance du mouvement et de l'interaction avec le monde ${ }^{39}$. Née de parents honorables qui la voulaient religieuse, la jeune fille est si attirée par l'apostolat des Ursulines qu'elle rejoint la communauté de Toulouse en 1607 contre le désir de sa famille. À cette date, la congrégation est encore libre d'aller et venir, et Rabonite est employée aux classes des externes ; elle s'occupe également d'activités apostoliques de par la ville, auprès des malades, des pauvres et des prisonniers. En 1616, elle devient religieuse cloîtrée comme ses camarades toulousaines, mais sa carrière continue de s'illustrer par l'action. En 1632, elle est envoyée à Béziers pour y prendre la tête d'une nouvelle branche en pays protestant; là, elle donne libre cours à son zèle missionnaire et s'efforce de dépasser les limites de sa propre claustration. Comme toute Ursuline doit le faire, elle ouvre un internat pour les pensionnaires aisées et un externat pour les filles de familles pauvres n'ayant pas les moyens de régler une pension. Néanmoins, elle choisit de ne pas se limiter à ce rôle et entreprend d'attirer de jeunes protestantes, des femmes de mauvaise vie et même des prostituées notoires

\footnotetext{
38. Linda LiERHEIMER, 'Female Eloquence and Maternal Ministry: The Apostolate of Ursuline Nuns in Seventeenth-Century France', thèse de doctorat non publiée, Princeton University, 1994, p. 236- 88.

${ }^{39}$. R. P. PARAYRe, Chronique..., op.cit., ii, p. 87-116.
} 
pour tenter, à force d'entretiens réguliers, de les ramener au catholicisme, ce qu'elle fait avec succès ${ }^{40}$.

Or, si bénéfiques soient-elles à l'Église catholique, ses actions ne sont pas conformes au rôle traditionnel des religieuses dans sa situation. Bien sûr, elle se met la population Protestante de la ville à dos quand elle entreprend de convertir des huguenotes; mais elle irrite aussi les catholiques en se mêlant de l'éducation des prostituées ou des voleuses, ce qui dépasse de loin son rôle d'Ursuline et qui correspond bien mieux à celui d'un prêtre. De surcroît, son succès témoigne d'une éloquence qui dérange d'autant plus qu'elle est supérieure aux savoir-faire requis par la définition contemporaine de la religieuse enseignante. Ainsi, les huguenots de la ville sont vite rejoints par de nombreux catholiques dans leur dénonciation des activités de cette catéchiste zélée. Françoise Rabonite est atteinte par la calomnie " la plus noire ", accusée de relations d'ordre sexuel avec son confesseur, d'illuminisme et de sorcellerie ${ }^{41}$. Abattue, elle quitte Béziers en 1653 et rentre à Toulouse où elle reste cloîtrée jusqu'à sa mort quelques mois plus tard. L'opinion générale à Béziers a condamné l'Ursuline qui n'avait point su s'en tenir au mandat habituel des religieuses de son Ordre.

La mission apostolique proposée par les Ursulines et les Dames anglaises transgresse de façon alarmante les décrets tridentins sur la clôture ${ }^{42}$. L'errance au féminin n'est jamais bien reçue, mais celle des religieuses est un scandale : en effet, puisqu'elles se réclament de l’Église, c'est bien la liberté de mouvement qui est à la source des principales difficultés tant des Ursulines françaises que des Dames anglaises. En 1615, Francisco Suarez (1548-1617) s'insurge contre les dites “ jésuitesses", soulignant leur absence de clôture et leur non-respect des décrets du

40. Ibidem, p. 103.

41 . Ibidem, p. 114

${ }^{42}$. Voir entre autres E. RAPLEY, The Dévotes..., op.cit. ; Ruth LIEBOwITZ, 'Virgins in the Service of Christ : The Dispute over an Active Apostolate for Women during the Counter-Reformation', in Rosemary RADFORD RUETHER (dir.), Women of Spirit, New York, Simon and Schuster, 1979, p. 131-152 et Linda LIERHEIMER, 'Redefining Convent Space : Ideals of Female Community among Seventeenth Century Ursuline Nuns', Proceedings of the Annual Meeting of the Western Society for French History, n 24, 1997, p. 211-20. 
Concile de Trente comme obstacles majeurs à leur réception en religion ${ }^{43}$. Il écrit : “ elles vont de par delà le pays sans rien de la bienséance du sexe féminin et causent l'ignominie de la religion catholique" ${ }^{44}$. En octobre 1622, le prêtre John Colleton ${ }^{45}$ exprime lui aussi ses inquiétudes :

“si [l'Institut] se confinait à ses cellules et à ses murs selon l'exemple des autres communautés religieuses, [il] mériterait peut-être nos louanges, mais quand il réclame une charge apostolique et erre sans aucune retenue de ci de là ... il s'expose sans doute à la censure et au reproche de bien des âmes pieuses ${ }^{~} 46$.

En outre, le bénédictin Sherwood trouve inacceptable que ces prétendues religieuses enseignent l'éloquence à leurs écolières et les encouragent à parler en public, à débattre de sujets religieux ou à prêcher. Comme les jésuites, elles mettent en scène des pièces de théâtre, activité qui témoigne d'une absence totale de réserve de la part des enseignantes ${ }^{47}$. La femme en mouvement ne connaît plus sa place dans la société, moins encore dans l'Église.

Il faut convenir que la réserve et l'effacement considérés essentiels à la femme, et a fortiori à la religieuse, échappent parfois à Mary Ward, toute absorbée du zèle de sa vocation. Son travail en Angleterre étant venu à la connaissance de l'archevêque de Canterbury, ce dernier ordonne un avis de recherche public, avec une description détaillée de la “jésuitesse "et une mise en garde selon laquelle cette femme " fait à elle seule plus de mal que six Jésuites "48. Ward, loin de se retirer dans l'anonymat, décide de rendre visite au prélat; elle part à pied pour Lambeth Palace, suivie de quelques compagnes, et grave son nom sur l'une des fenêtres du palais à l'attention de l'archevêque qui s'était absenté ce jour-là. Que cet épisode soit réel ou qu'il provienne de l'imagination d'un chroniqueur quelconque importe peu : il n'en

\footnotetext{
${ }^{43}$. H. PETERS, Mary Ward ..., op. cit., p. 154-57

44. ABC, C1, Letters against the Jesuitesses, f. 297.

${ }^{45}$. Pendant le conflit entres Jésuites et prêtres séculiers de la mission anglaise, John Colleton forme l'Association du clergé d'Angleterre, corps indépendant chargé de réguler les affaires du clergé séculier. A cause de cette initiative, il est plus tard accusé d'aggraver le schisme entre séculiers et Jésuites et suspendu de ses fonctions. Voir Ethelred TAUNTON, The History of the Jesuits in England, 1580-1773, Londres, Methuen, 1901, p. 256-57.

${ }^{46}$. ABC, $\mathrm{C} 1, \mathrm{~J} 3$, translation of documents re. Jesuitesses.

${ }^{47}$. ABC, B 45, J. GRISAR, The First Accusations..., op. cit., p. 61.

${ }^{48}$. ABC, B12, A Briefe Relation...., op. cit., f. 21.
} 
reste pas moins représentatif de l'impudente hardiesse attribuée à la fondatrice par ses détracteurs comme par ses disciples.

Ce défaut de retenue est interprété par le clergé comme un acte de rébellion, et par les historiens d'aujourd'hui comme une revendication féministe avant l'heure. En effet, à une époque où l'Église a besoin de toute l'aide qu'elle peut mobiliser pour augmenter le nombre de ses fidèles, il semble évident à Mary Ward qu'une congrégation travaillant à catéchiser la population féminine sans les restrictions de la clôture représenterait un avantage considérable. Elle ne partage aucunement les opinions androcentriques qui prévalent alors; elle croit la femme tout à fait capable de mener cette tâche à bien et de rester constante même au cœur d'une mission hasardeuse, de ville en ville, de pays en pays. Elle déclare un jour à ses consœurs : “Je prie Dieu que les hommes comprennent cette vérité : que les femmes, si elles le veulent, peuvent atteindre la perfection, et que s'ils cessaient de nous faire croire que nous ne pouvons rien faire, et que nous ne sommes rien que de pauvres femmes, nous pourrions réaliser bien des prouesses $" 49$. La force de sa conviction, alliée à son peu de diplomatie, finissent de convaincre les autorités de son obstination à nier les normes de l'époque. Tout à son dévouement envers la contre-réforme, Mary Ward oublie que, pour le clergé, les rôles masculins et féminins ne sont guère interchangeables : une mission hautement respectée chez les hommes peut donc constituer une entreprise inacceptable chez les femmes. Cette erreur s'avère fatale et alors que les Ursulines françaises prospèrent, les filles de Mary Ward voient le Pape Urbain VIII prononcer la suppression définitive de leur Institut et condamner leur fondatrice comme rebelle et hérétique ${ }^{50}$.

L'Institut de Mary Ward, qui tente d'imiter les jésuites le plus fidèlement possible, est brutalement supprimé en 1631. La déclaration papale dénonce “la prétendue congrégation des jésuitesses " comme dangereuse et devant être éradiquée à

\footnotetext{
49. ABC, B17, f. 3.

${ }^{50}$. ABC, boite d'archives $n^{\circ} 1$, pièce 2 , Bulle papale.
} 
tout prix. L'un des motifs principaux est bien sûr le manque de clôture des Dames anglaises et ce qu'Urbain VIII appelle leur liberté “ d'aller et venir comme bon leur semble et, sous l'apparence de promouvoir la sauvegarde des âmes, d'entreprendre d'autres travaux mal seyants à la faiblesse de leur sexe et de leur entendement, ainsi qu'à la modestie féminine " ${ }^{51}$. Cependant, de façon paradoxale, cette suppression est comme une libération pour la mission active de l'Institut; à l'abri des objections canoniques, les Dames anglaises privées de leur statut clérical peuvent désormais laisser libre cours à leur zèle sur le terrain. Bien sûr elles sont toujours, en tant que femmes en mouvement, soupçonnées de mœurs légères et sujettes à la raillerie populaire, mais elles ne représentent plus de danger pour le clergé. Les prêtres qui les voyaient comme rivales implicites ne les considèrent plus que comme laïques réunies autour d'une mission pieuse. Grâce à ce statut inoffensif, elles sont plus libres qu'elles ne l'étaient alors qu'elles réclamaient la reconnaissance religieuse.

Le succès des Ursulines s'explique, au contraire, par leur soumission à la clôture grâce à laquelle elles gagnent le respect du peuple et de l'Église. L'histoire des premières années de l'Ordre indique que si les congrégées avaient persisté dans leur forme initiale, indépendante et sans clôture, mais réclamant le statu de religieuses, elles auraient sans nul doute connu un sort similaire à celui de l'Institut de Mary Ward. Mais les murs du couvent les protègent. Cloîtrées, elles ne peuvent usurper les attributs masculins des missionnaires et prédicateurs de la contre-réforme. La formule ursuline est cependant plus subtile que la traditionnelle vie religieuse, puisque les sœurs ne renoncent pas à leur vocation active et pédagogique. Soumises à l'immobilité physique qu'implique leur statut, elles sont en contrepartie autorisées à recevoir les externes au sein du couvent et à donner des leçons de doctrine aux grilles de l'église. C'est donc le mouvement qui vient à elles sous la forme des externes et des foules qu'elles enseignent. Par cette simple manœuvre, les Ursulines restent fidèles à leur vocation évangélique sans risquer d'alarmer le clergé. Cette subtile redéfinition de la clôture monastique suffit à garantir la sécurité de leur mission. 
La liberté de mouvement, si créatrice et si précieuse chez les clercs réguliers masculins, demeure une impossibilité chez leurs émules du "sexe le plus dangereux et le plus faible " ${ }^{52}$. Pour que ses activités de par le monde soient légitimes, la femme se doit de demeurer séculière. Au cœur de ce paradoxe de l'Église de la contreréforme se trouve le stigmate de la femme en mouvement. La mobilité spatiale est à peine acceptable lorsqu'elle est laïque, mais semble anathème à la définition de la femme d'Église : on ne saurait avoir de religieuses errantes. Ainsi les promesses de l'aube du XVII ${ }^{\mathrm{e}}$ siècle restent-elles lettre morte pour ces dernières ; les déplacements demeurent un privilège masculin ordonné par les décrets du Concile de Trente et confirmé par la papauté au moment même de la plus fervente vague de dévotion féminine de l'histoire moderne.

Laurence Lux-Sterritt

LERMA

Université de Provence I

29, Avenue Robert Schuman

13621 Aix-en-Provence Cedex 01

sterritt@up.univ-aix.fr

${ }^{52}$. R. P. Parayre, Chronique..., op. cit., i, p. 58. 